\title{
Convidar a las almas, convidar a la Tierra. Lógicas rituales y categorías de relación entre seres en la Argentina andina
}

Inviter les âmes, inviter la Terre. Logiques rituelles et catégories de relation entre les êtres en Argentine andine

Inviting the Souls, inviting Mother Earth. Ritual logics and categories of relations between human and non-human beings in Andean Argentina

\section{Daniela Salvucci}

\section{OpenEdition}

Journals

Edición electrónica

URL: http://journals.openedition.org/bifea/7995

DOI: 10.4000/bifea.7995

ISSN: 2076-5827

Editor

Institut Français d'Études Andines

Edición impresa

Fecha de publicación: 1 agosto 2016

Paginación: 289-305

ISSN: 0303-7495

Referencia electrónica

Daniela Salvucci, « Convidar a las almas, convidar a la Tierra. Lógicas rituales y categorías de relación entre seres en la Argentina andina », Bulletin de l'Institut français d'études andines [En línea], 45 (2) I

2016, Publicado el 08 agosto 2016, consultado el 05 noviembre 2020. URL : http://

journals.openedition.org/bifea/7995; DOI : https://doi.org/10.4000/bifea.7995

\section{(c) (†) $\odot$}

Les contenus du Bulletin de l'Institut français d'études andines sont mis à disposition selon les termes de la licence Creative Commons Attribution - Pas d'Utilisation Commerciale - Pas de Modification 4.0 International. 


\title{
Convidar a las almas, convidar a la Tierra. Lógicas rituales y categorías de relación entre seres en la Argentina andina
}

\author{
Daniela Salvucci*
}

\begin{abstract}
Resumen
El ensayo propone un análisis comparativo de los rituales del «pago a la Tierra» de agosto y del «día de las almas» de noviembre que se realizan en el altiplano andino de Jasimaná (provincia de Salta, noroeste de Argentina). Utilizando como base una serie de descripciones etnográficas, se analizarán tanto las lógicas de los ritos (el convite, la ofrenda, el sacrificio, la separación), como las categorías émicas que expresan y producen las relaciones rituales entre humanos y no humanos. Se hará referencia a la teoría de los sistemas ontológicos (Descola, 2005) para comprender dichas relaciones entre seres que, si bien interconectados, se mantienen distintos unos de otros.
\end{abstract}

Palabras clave: rituales, ontologías andinas, noroeste argentino

\section{Inviter les âmes, inviter la Terre. Logiques rituelles et catégories de relation entre les êtres en Argentine andine}

\section{Résumé}

Cet article présente une analyse comparative du rite appelé « paiement à la Terre », qui a lieu en août, et de celui du « jour des âmes », réalisé en novembre, sur le plateau andin de Jasimaná (province de Salta, nord-ouest de l'Argentine). En partant de descriptions ethnographiques, on analysera aussi bien les logiques des rites (l'invitation, l'offrande, le sacrifice, la séparation), que les catégories « emic » qui expriment et produisent les relations rituelles entre humains et non-humains. On fera référence à la théorie des systèmes ontologiques (Descola, 2005) pour mieux comprendre ces relations entre des êtres qui, même s'ils sont interconnectés, restent bien distincts les uns des autres.

Mots-clés: rituels, ontologies andines, Nord-Ouest Argentin

* Investigadora posdoctoral, Universidad Nacional de Catamarca. Casa de Posgrado, calle Rivadavia 265, San Fernando del Valle de Catamarca, Argentina. E-mail: salvuccidana@gmail.com 


\title{
Inviting the Souls, inviting Mother Earth. Ritual logics and categories of relations between human and non-human beings in Andean Argentina
}

\begin{abstract}
The article presents a comparative analysis of two rites that take place in the Jasimaná Highland (province of Salta, North Western Argentina): the "payment to the Earth" in August and the "day of the Dead' Souls" in November. Using ethnographic descriptions, it focuses on both the ritual logics (as the invitation, the offering, the sacrifice, and the separation) and the emic categories that express and produce ritual relations between humans and non-humans. The theory of ontological systems (Descola, 2005) helps us to understand relations between diverse beings that remain different, although connected to each other.
\end{abstract}

Keywords: rituals, Andean ontologies, Northwestern Argentina

\section{INTRODUCCIÓN}

Los pastores y pastoras que habitan el altiplano de Jasimaná (3300-3800 msnm), provincia de Salta, noroeste andino de Argentina, utilizan la comida y otros bienes de consumo alimentario, como las hojas de coca y el alcohol, de manera ritual. En ocasiones específicas ofrecen todo esto a la «Pachamama-Santa Tierra» en el mes de agosto y a los muertos por el día de las almas, entre el 1 y 2 de noviembre. A lo largo de los caminos los habitantes pueden «convidar» hojas de coca a la Pachamama, de la misma manera en que se convidan a las personas que pasan caminando, pero enterrando la coca o soplándola en el aire. En cambio, el rito llamado «pago», que se realiza en agosto, implica un «sacrificio», por ejemplo el entierro de un feto animal como ofrenda a la Tierra. Mientras que, por el día de las almas, se ofrecen en una mesa especial los muchos platos de comida que se cocinan para los difuntos. Pese a que este ritual convida a los muertos en el espacio familiar de la casa y de la cocina, junto con otros parientes y vecinos, los vivos y los muertos quedan separados. Además, los vivos deben apurarse y despedir a los difuntos el 2 de noviembre al mediodía, enterrando afuera de la casa la comida reservada para ellos.

Luego de una breve presentación del altiplano de Jasimaná y de sus habitantes, se brindarán descripciones de los ritos del pago y del día de las almas utilizando datos etnográficos que he producido en 2011 y 2015, en un marco metodológico interpretativista (Geertz, 1973). Durante los trabajos de campo, la técnica de la observación participante ha sido dirigida a la interpretación de las prácticas locales y a la traducción de las categorías émicas, es decir producidas por los mismos actores sociales desde su universo cultural. A partir de las descripciones etnográficas, el análisis destacará y comparará las lógicas rituales del pago y del día de las almas, seleccionando aquellas categorías de relación entre seres humanos y no-humanos asociadas a los ritos. Finalmente, haciendo referencia a la teoría de 
los sistemas ontológicos y al concepto de analogía elaborados por Descola (2005), se dibujará un esquema abstracto de las continuidades y diferencias ontológicas entre seres relacionados ritualmente, indicando aquellas prácticas y categorías que permiten cierto «acercamiento ontológico» entre ellos. Principalmente, exploraremos hasta qué punto las prácticas del «convidar» y las categorías émicas de parentesco, basadas en el compartir la cocina y la comida (Weismantel, 1988; Harvey, 1998), puedan incluir a los muertos, además de discutir su asociación con otros seres no humanos muy poderosos y hasta peligrosos como la Pachamama. La finalidad del texto, de hecho, es reflexionar sobre las relaciones establecidas por los ritos y las características de los seres relacionados.

\section{JASIMANÁ}

El altiplano de Jasimaná se encuentra entre 3300 y 3800 msnm aproximadamente, en la Cordillera andina meridional, en el suroeste de la provincia argentina de Salta. Sus habitantes son miembros de la Comunidad Indígena Diaguita-Calchaquí1.

En los años 1980, alrededor de las escuelas primarias recién instaladas, se formaron tres pequeñas aldeas: Río Grande, El Arremo y Pampallana, que cuentan con una capilla católica y un puesto de salud con un enfermero residente. En las aldeas no hay agua corriente y muy pocas casas tienen paneles solares o motores con gasolina para la electricidad; tampoco hay red telefónica ni conexión a internet.

En cada una de las aldeas viven aproximadamente 250 habitantes (datos Agentes Puestos Sanitarios, comunicación personal, 2011), la mitad de los cuales son menores de edad. Sin embargo, el patrón de residencia de las unidades domésticas (o grupos domésticos) es «múltiple» (Gil Montero, 2004), en el sentido de que los diferentes miembros de las unidades habitan desplazándose entre dos o tres casas: la casa en la aldea, la casa de campo y el puesto. Según la expresión local, la unidad doméstica junta a los que «tienen la cocina aparte», pero en las aldeas los que comparten la cocina viven en complejos habitacionales familiares, compartiendo los patios con otras unidades domésticas de parientes. Los parientes son definidos por la categoría émica «ser familia» que implica el «reconocerse», en tanto que parientes, y el «respetarse» (Salvucci, 2013). En las aldeas, las casas más recientes están hechas de adobe o ladrillos y techo de chapa.

En cambio, en el campo, cada grupo doméstico tiene su casa compuesta por piezas (cocina, dormitorios y almacenes) y los corrales para los rebaños. Las piezas son de piedra y barro, con puertas de madera de cardón y techo de ramas, torta

1 Se trata de una comunidad indígena con personería jurídica de «pueblo originario». Los habitantes de las aldeas de Río Grande, El Arremo y Pampallana pertenecen a la comunidad por inscripción (además de los maestros y los enfermeros residentes temporales). La comunidad ha sido reconocida oficialmente por el INAI (Instituto Nacional de Asuntos Indígenas del Ministerio de Desarrollo Social) gracias a la nueva constitución nacional argentina de 1994. Esta admite la preexistencia histórica de los muchos pueblos indígenas del país, otorgándoles derechos políticos y económicos específicos, en el marco de revisión crítica del proceso de construción nacional basado en la represión, invisibilización y discriminación de los grupos indígenas y de sus culturas. 
de barro y paja. Estas casas se encuentran dispersas en el altiplano, bastante lejos una de otra, pero ubicadas cerca de vertientes o arroyos.

La mayoría de los habitantes de Jasimaná son «criadores» de ganado, como muchos se definen, pero se considera al pastoreo de cabras, ovejas y llamas una ocupación femenina, en tanto que los varones son agricultores y criadores de ganado vacuno, o se dedican al comercio. Para ello, viajan en camioneta, durante 3-4 horas, hacia el valle para abastecer de mercaderías a los pequeños almacenes de las aldeas. De acuerdo a los relatos de vida recolectados, antes de la construcción del camino de tierra para los autos, los hombres de cada grupo doméstico realizaban largos viajes de intercambio y compra-venta de bienes con caravanas de burros de carga. Iban hacia la finca de Pucará, que linda al norte con Jasimaná, para abastecerse de maíz y verduras, y bajaban hacia los valles Calchaquíes para vender sus productos, como carne, charqui (carne deshidratada en quechua2), hilados, hierbas que se usan como remedios caseros (yuyos). A cambio conseguían vino, trigo, fruta seca en los mercados de Cafayate, Santa María y Angastaco.

Hoy en día, muchos de los jóvenes, sobre todo varones, emigran de forma temporal y estacional pero también permanente, a los pueblos del valle y a las ciudades del sur del país para trabajar como mano de obra, generalmente en construcción o en fincas, mientras que las mujeres son contratadas como empleadas domésticas o cocineras.

Según las pautas de residencia de los grupos domésticos, un adulto se queda en la casa de la aldea durante la semana para cuidar a los niños (hijos, sobrinos y/o hermanitos) que van a la escuela, al mismo tiempo que un pastor principal, generalmente una mujer, trabaja en la casa de campo, cuidando los rebaños. Estos son Ilamados «la hacienda» y reúnen a los animales de todos los miembros de la unidad doméstica. Sin embargo, la pertenencia de las reses es individual; hasta los niños poseen sus animales. También los miembros del grupo doméstico que han dejado el altiplano en forma temporal o permanente pueden tener su «tropa» de animales en el rebaño del grupo y volver a Jasimaná para participar en la esquila de la lana o en el ritual anual de la «señalada», que consiste en marcar los animales jóvenes con cortes en las orejas, de forma distinta para cada dueño.

Cuando no hay escuela, cada fin de semana y durante las vacaciones, los que residen en la aldea se desplazan a la casa de campo recomponiendo así la unidad doméstica. A veces, solo los chicos mayores van para ayudar o para hacer el cambio de turno con la pastora principal (generalmente su mamá, abuela o hermana) que cuida la hacienda, permitiéndole así regresar a la aldea o viajar hacia el valle.

Además, la pastora principal se desplaza también según la temporada entre la casa de campo y el puesto, quedándose en la primera, ubicada en las tierras bajas del altiplano, durante la temporada húmeda, desde diciembre hasta mayo, de manera que los rebaños aprovechen del pasto fresco recién brotado después de las lluvias

2 Si bien los habitantes de Jasimaná hablan castellano, también utilizan muchas palabras quechuas y unas cuantas aimaras, generalmente castellanizadas, sobre todo en relación con enfermedades, prácticas de cura, plantas y animales. Además, muchos topónimos son palabras kakanas, de origen diaguita, como por ejemplo «Jasimaná» que significa «pedregal» (Jiménez Sánchez-Mariscal, 2014: 24). 
Lógicas rituales y categorías de relación entre seres en la Argentina andina

de enero y febrero. Durante la temporada seca, cuando el pasto de abajo ya se ha acabado y solo queda la vegetación permanente de las altitudes mayores, la pastora se traslada con los rebaños hacia las tierras altas, donde vive en un puesto (otra casa hecha con piezas de piedra y corrales pero más pequeña que la de campo).

Finalmente, cabe señalar que el manejo de los rebaños se realiza gracias al pastoreo extensivo, es decir que los animales son sacados del corral por la mañana, o «despachados» según la expresión local, para que se alejen en búsqueda de pasto ${ }^{3}$. Por la tarde las reses vuelven solas al corral, aunque en el caso de las ovejas las pastoras tienen generalmente que ir a buscarlas, ayudadas por los perros, utilizando la honda a lazo para echar piedras que asustan y empujan a los animales a volver a la casa.

Los animales de la hacienda y los «ayudantes-perros» ${ }^{4}$ participan en muchos de los rituales que los pastores realizan, como las señaladas y el pago a la Tierra. Dichos ritos involucran al grupo doméstico y a los parientes. Entre los parientes están tanto los vivos como los muertos, o sea «las almas», una categoría que indica también a los difuntos en general. Como muestran los relatos etnográficos que se presentan a continuación, los rituales realizados en Jasimaná relacionan a seres diferentes: personas, animales, almas, santos y virgencitas (pequeñas estatuas guardadas en las casas y llevadas en andas en procesión) y la Pachamama-Santa Tierra.

\section{1. El pago a la Tierra}

El «pago a la Tierra» se realiza en agosto, tanto a nivel familiar como comunitario. Cada grupo doméstico hace el pago en la casa de campo, cerca del corral principal, aunque muchos lo celebran en la casa de la aldea. Sin embargo, en los últimos años se organiza también un pago en la escuela donde participan los alumnos, unos cuantos padres y los maestros. Si bien este acto es dirigido por el director de la escuela, son las señoras de la aldea que trabajan en la escuela como cocineras o encargadas de la limpieza, a la vez pastoras indígenas, las que guían la secuencia ritual. En este caso, el pago se realiza cerca de la escuela, con la presencia de la bandera indigenista de la Wiphala, la bandera de la región de Salta y la bandera nacional.

Además, desde el año 2004, la comunidad Diaguita Calchaquí realiza un «despacho» a la Pacha el 31 de agosto como cierre del mes dedicado a la Pachamama. El despacho es dirigido por el cacique, que lleva la chuspa (bolsa) ritual y la faja de colores de la Wiphala, y tiene lugar en la casa comunitaria de la aldea, con las banderas, incluyendo a todos los habitantes y contando también con la participación de los maestros.

3 Las tierras de pastoreo de cada unidad doméstica no son divididas por corrales o pirkas, pero los confines se conocen y dependen del principio del «respeto» de las divisiones de tierra establecidas por el último contrato patronal.

4 Flores Ochoa (1977: 231-232) afirma que en la sierra peruana al perro no se le considera un animal sino un ayudante del runa (hombre en quechua). 
Pese a las variaciones de dichas ceremonias, todo ritual nombrado implica una ofrenda a la Pachamama en forma de entierro, un almuerzo en común, la presencia de la hacienda y la construcción de la apacheta (un altar consagrado a la Pachamama hecho de piedras blancas apiladas).

El pago a la Tierra que cada familia realiza en su casa empieza por la mañana, temprano, generalmente el 1 de agosto, aunque todo el mes está dedicado a la Pachamama.

Siendo todavía de noche, los miembros de la unidad doméstica recolectan la basura de la casa y la queman en uno o varios fuegos encendidos alrededor de ella. Al mismo tiempo preparan un sahumerio con hierbas del monte y a veces lana de llama negra, al que también pueden agregar el sahumerio boliviano: una mezcla de inciensos, papel de colores picado, cigarrillos y alasitas (miniaturas, de la palabra aimara «alasitas» que significa «cómprame») de billetes de dólares, que se compra en los almacenes de las aldeas. Después de sahumar la casa, todos toman un té de ruda, ajo y alcohol, en tres tragos. Se friccionan los brazos y las manos con alcohol puro o mezclado con un remedio de hierbas y se atan al cuello, muñecas, cintura y tobillos el «hilo zurdo», previamente mojado en el té de ruda y alcohol, ayudándose unos a otros. El hilo zurdo es un cinto de hilos de dos colores (por ejemplo negro y blanco o verde y rojo) hilados en sentido contrario (por este motivo se le llama «zurdo»). Los atados de hilo zurdo no se sacan hasta el final del mes cuando son enterrados como ofrenda a la Pachamama durante la ceremonia del despacho organizada por la comunidad.

Al amanecer, los que comparten la misma hacienda, es decir los miembros del grupo doméstico y unos parientes más, se desplazan de la casa al corral para sahumarlo con los animales adentro. Se atienden las tareas diarias del rebaño, como hacer mamar a los cachorros «guachos» (huérfanos o botados por su mamá) de otra hembra con leche, ya que en esta época del año los animales han dado crías. La pastora principal procede al corte de la cola de los «corderitos», ayudada por los demás participantes. Primero, se preparan hilos espesos de lana gruesa teñida, en doble color; luego, los chicos, jugando y corriendo, agarran a los corderos, los «chimpean», es decir que les atan un cinto de hilos de lana gruesa al cuello y a una patita, y los llevan para que la pastora principal con una o un ayudante les practique el corte de la cola con un cuchillo y eche tierra a la herida para que no sangre tanto. Las colas de los corderos se guardan en una bolsa para luego ser ofrendadas a la Pachamama. Otros animales, sobre todos los jóvenes y los reproductores, son «floreados», es decir que se les ponen aritos de lana gruesa de color en las orejas empleando una aguja, y también «chimpeados». Finalmente, todos los animales de la hacienda son despachados y salen del corral buscando pasto.

Alrededor del mediodía, mientras se va terminando de cocinar la comida para el almuerzo, se destapa el agujero para la ofrenda a la Pachamama, desarmando la apacheta realizada el año anterior cerca del corral. Debajo del apilamiento de piedras blancas, una piedra ancha y chata tapa el agujero: hay que removerla y excavar un poco, sacando tierra con la pala para abrir una especie de boca de la Pachamama-Santa Tierra. Alrededor del agujero se van disponiendo las bebidas: 
gaseosas, vino y pequeñas botellas de aguardiente; las cajitas de cigarrillos y las bolsas con las hojas de coca. Cuando se llevan las ollas y bandejas con la comida, todos los participantes nos ubicamos alrededor del agujero para realizar la ofrenda a la Pachamama: uno tras otro echamos en el agujero las bebidas dibujando una cruz con el chorro, cantidades de hojas de coca y cigarrillos encendidos clavándolos en horizontal adentro del agujero. El almuerzo (carne asada u horneada, sopa o locro, papas criollas y mazamorra) se consume al lado del agujero, donde se coloca buena parte de la comida antes de empezar a probar cualquiera de los platos. Se dice que esto es «compartir con la Pacha» y las madres sugieren a los chicos que «la mitad sea para la Pacha y la mitad para uno». Como la Tierra es muy «golosa», después de darle mazamorra (una sopa dulce de maíz con duraznos secos y azúcar) como postre, se le brinda abundante cantidad de dulces, fruta seca, fruta fresca (plátanos, naranjas, manzanas), queso, galletitas, caramelos, pero nunca se le ofrece sal pura. Al final, se vierten adentro todas las bebidas y todo lo que queda, incluso más cigarrillos y hojas de coca. Por último, se echan en el agujero las colas cortadas de los corderos, chimpeadas con hilos de lana gruesa teñida, pidiendo a la Pachamama salud para los miembros de la familia y para la hacienda. Al final, se tapa el agujero con la piedra larga y chata y se arma la apacheta, primero chimpeando las piedras blancas del año anterior y las nuevas que se recolectaron para la ocasión. Cada uno pone sus piedras chimpeadas formando una montañita, a veces aclarando que «las piedras son sus corderos y chivos». Si entre los participantes hay copleros con caja, estos, dando vueltas alrededor de la apacheta, «echan coplas» o le «gritan a la Pacha», que, según dicen, disfruta de la alegría de la gente en este día.

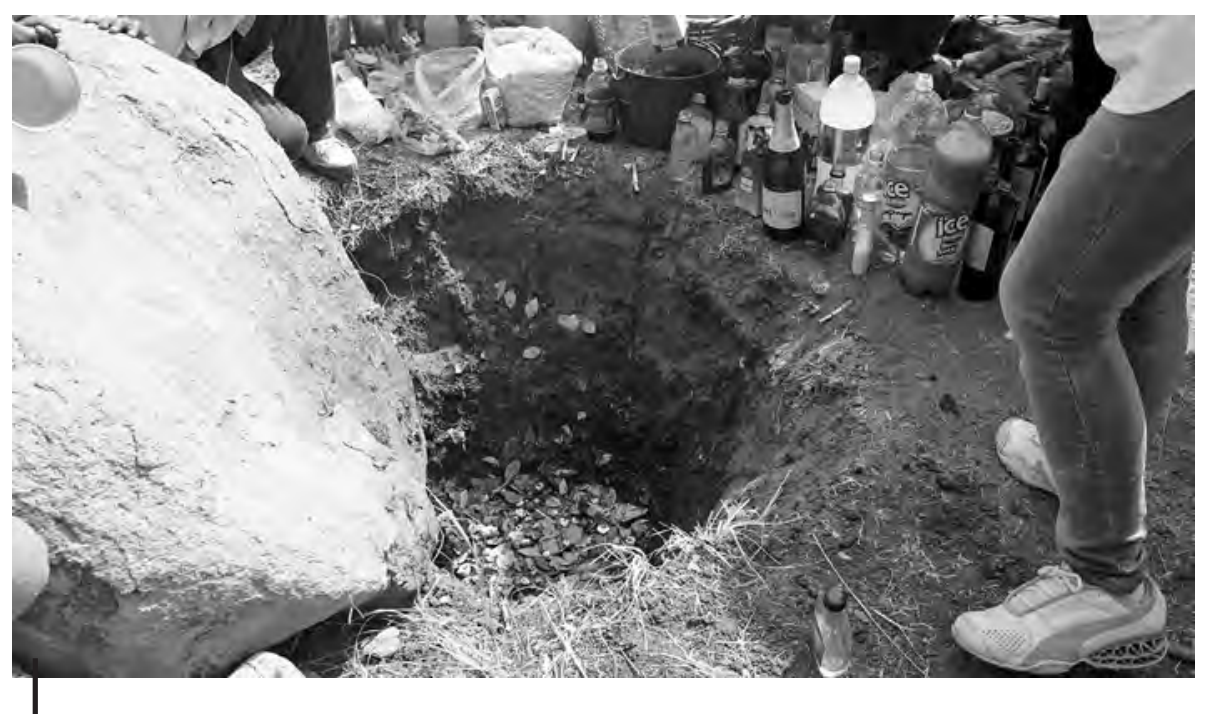

Figura 1 - Ofrenda a la Pachamama en forma de entierro realizada por los alumnos de la escuela (Río Grande de Jasimaná, 3 de agosto de 2015)

(C) Daniela Salvucci 
También se puede ofrecer a la Pachamama, enterrándolo, un feto animal o directamente un cordero sacrificado, «bien floreado y chimpeado», porque «a la Pacha le gustan los colores».

\section{2. El día de las almas}

Se considera que las almas de los muertos vuelven a su casa y visitan a sus parientes cada año los días 1 y 2 de noviembre. Los miembros de la unidad doméstica, junto con parientes y vecinos, se reúnen en la casa de campo o en la casa de la aldea para esperarlas. Ya desde la víspera, por la tarde del 31 de octubre, cada jefe o jefa de hogar lleva hacia la casa las andas con sus santos y virgencitas atados uno a otro con cintos de colores para que no se caigan o rompan. De hecho, muchos dejan sus andas en la capilla cuando llega el «padrecito» (el cura) del pueblo del valle a visitar la aldea, para la fiesta patronal en septiembre, y ahí las alumbran con velas. Para el día de las almas cada familia lleva sus andas hacia la casa de campo o de la aldea, formando una pequeña procesión con los chicos que tocan el bombo y llevan banderas de la nación y de la Iglesia hechas en casa.

A partir de la mañana del día 1, se carnea un animal (una llama, por ejemplo) y se arma la «mesa de las almas»: una linda mesa con mantel y una cruz en donde de a pocos se van poniendo todas las bebidas y todos los platos preparados a lo largo del día para los difuntos, esperando su llegada. Se sirve primero el desayuno, ofreciendo en la mesa el más bonito mate con bombilla, el bollo (pan casero), las tortillas y la ulpada (una bebida de harina). Antes de poner los platos en la mesa de las almas, se separa la parte que será consumida por los vivos que participan en el evento, los que ocupan otra mesa más pequeña y ordinaria. Antes de comer, cada uno separa el primer bocadillo en un tacho dedicándolo a las almas.

Para el almuerzo, y luego para la cena, se cocina una gran cantidad de platos diferentes y sabrosos: locro, picante, asado, papas criollas, habas, mote, morcillas, empanadas, buñuelos con huevos de suri (ñandú), anchi (una sopa dulce de harina de maíz), mazamorra con pelones (duraznos secos), higos secos y uva pasa. Se ofrece vino patero traído de Angastaco y también hay quien prepara chicha de maíz y aloja de algarroba. De hecho, es el día en el que más se cocina. Sobre todo hay que preparar la comida según «el estilo de los de antes», es decir a la manera de los antepasados, y cocinar lo que a ellos, a las almas, solía gustarles: comida bien casera, cocinada en ollas de barro y servida en bandejas de madera, con cubiertos de madera, al estilo antiguo. También se considera mejor moler el maíz en el mortero, como hacían los abuelos y los padres. En la mesa no deben faltar cigarrillos y bolsas llenas de hojas de coca. En el tacho que va llenándose de comida, separada por los participantes al momento de comer cualquier nuevo plato, también se ponen hojas de coca y se dejan consumir cigarrillos encendidos.

Antes de cenar se realiza la «desatada» de los santitos y virgencitas. Uno tras otro, todos los participantes desatan una estatua de las andas, ofreciendo a cambio algo de plata, mientras que uno de la familia toma nota de su nombre y de la 
cantidad de dinero donada. Cada estatua desatada es besada por los presentes, puesta en una habitación junto con las demás y alumbrada. Cuando todos los santos y las virgencitas son guardados y las andas con sus flores, espejitos y tulles son desarmadas por completo, recién se sirve la cena, ofreciendo primero la comida en la mesa de las almas. Luego, alguien de la unidad doméstica lee los nombres y apellidos de las almas escritos en papelitos. Si participa gente de otra unidad doméstica, ellos también escriben los nombres de sus almitas para que sean leídos en voz alta. Además, se recuerda a los «angelitos» de un grupo doméstico, es decir los hijos muertos cuando niños. Luego de «nombrar a las almas», se leen los nombres y el monto de plata donada por cada uno de los participantes en la desatada y se «paga» a los que donaron con unas cuantas roscas dulces en relación con la cantidad de plata que dieron. Por la noche se sigue esperando a las almitas, a lado de la mesa alumbrada con velas hechas de pulpa de cardón y grasa de oveja. Por la mañana del día 2, se cocina el almuerzo y se ofrece aún más comida, pero alrededor del mediodía se separa todo lo que está en la mesa, volcando la mitad de cada plato en un tacho grande, agregando también todos los bocadillos de comida que se fueron acumulando en el tacho pequeño. Se dice que hay que «despachar a las almas». Los que participaron en la velada, bastante apresurados, se desplazan afuera pero cerca de la casa y destapan el agujero en donde enterraron la ofrenda para las almas el año anterior. Todo el contenido del tacho se vuelca en el agujero, echando también las bebidas, las hojas de coca y los cigarrillos. Finalmente se tapa el agujero con una piedra grande y se clava por encima una cruz; luego, todos los participantes vuelven a la casa para almorzar juntos.

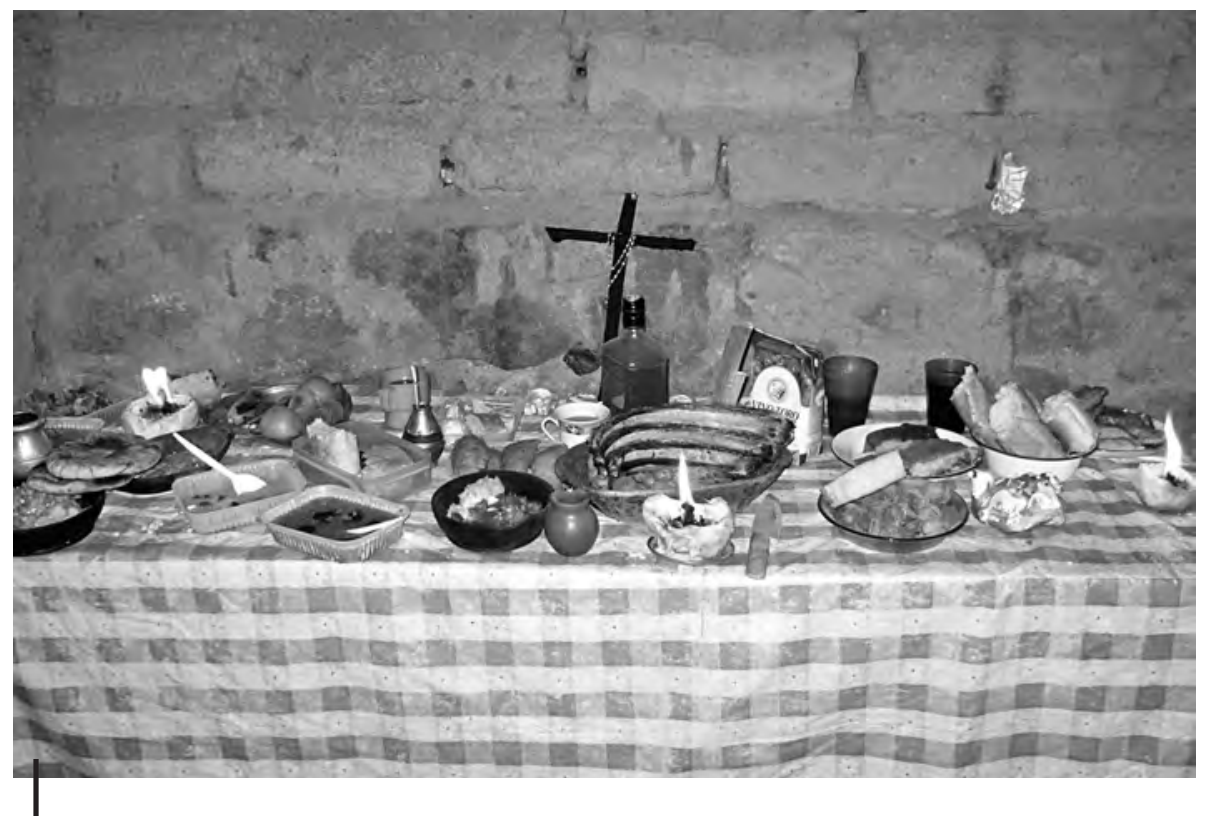

Figura 2 - Mesa de las almas (Río Grande de Jasimaná, 1 de noviembre de 2011)

(C) Daniela Salvucci 


\section{CATEGORÍAS DE RELACIÓN Y LÓGICAS RITUALES}

Con «categorías de relación» me refiero a las categorías émicas asociadas a las prácticas que expresan relaciones entre los seres incluidos en los ritos. Sin embargo, no se trata solo de maneras de decir, sino de expresiones involucradas en las acciones, textos incorporados al contexto.

El análisis sincrónico de dichas categorías y de las secuencias de acción ritual descritas del pago a la Tierra y del día de las almas nos permitirán interpretar los ritos por medio de categorías interpretativas sintéticas para definir las diferentes «lógicas» de acción y relación ritual entre los seres.

Una de las categorías émicas asociadas al pago a la Tierra es el «hambre». Se dice que en agosto la Tierra está «hambrienta» y que es «delicada como una mujer embarazada, a punto de parir» y que, por lo tanto, es muy peligroso andar caminando, a mula o a caballo y hasta manejar la camioneta. Cuando la Tierra tiene hambre puede «comer» humanos: no solo se dice que en agosto es muy probable caerse, lastimarse o asustarse, es decir tener un accidente $y / o$ enfermarse, sino que mucha gente también fallece en esta época. El «hambre» de la Tierra implica una relación de peligro y «cuidado» respecto de ella. El «cuidado» es sobre todo un cuidado ritual, incluye protegerse ritualmente, hacer ofrendas a la Pachamama a lo largo de los caminos y realizar el pago: especialmente en agosto, cuando más «hambre» tiene, hay que «dar de comer a la Tierra».

Las categorías émicas del «hambre», del «cuidado» y del «dar de comer a la Tierra» están asociadas al pago y a las relaciones entre personas, hacienda y Pachamama que se producen y expresan en este rito.

Como resulta del relato etnográfico, la secuencia del pago incluye: una limpieza ritual de la casa (quema de la basura, sahumerio de las piezas y del corral) y de las personas (friccionarse, tomar té de ruda); un acto de autoprotección (atarse el hilo zurdo); una ofrenda de sangre (en nuestro caso el corte de las colas de los corderos) y una ofrenda de bienes de uso también alimentario en forma de entierro, acompañada por el pedido de protección; finalmente, la construcción de un altar (estableciendo una relación entre piedras y animales). Se trata básicamente de una lógica de «protección», tanto de personas como de animales, y de una lógica de «ofrenda-sacrificio».

Protección remite a la autoprotección que se realiza atándose el hilo zurdo, más el pedido de protección que el pastor o la pastora principal dirige hacia la Pachamama pidiendo para la salud e inclusive para la reproducción de los miembros de la familia, humanos y animales. De hecho, se pide a la Pacha no solo que «el zorro y el león no sean dañinos y no perjudiquen a la hacienda», sino también que haya «una buena cosecha de corderos», una multiplicación de la hacienda. Esto establece una conexión entre el pago y el ritual de la «señalada» de los animales, cuya finalidad explícita es el «multiplico» (Bugallo, 2014), es decir la reproducción del rebaño. En ambos ritos los animales son floreados y chimpeados, así como 
Lógicas rituales y categorías de relación entre seres en la Argentina andina

son chimpeadas las piedras de la apacheta que se levanta al final, asociadas a los animales deseados, tanto durante el pago como en la señalada.

La lógica de la ofrenda en forma de entierro, que también encontramos en el rito del día de las almas, implica, en el caso del pago, un «sacrificio». El «dar de comer a la Tierra» no se refiere solo a los bienes alimentarios-rituales (hojas de coca, alcohol y cigarrillos) y a los bienes alimentarios cocinados, además de los muchos dulces, sino que incluye también una ofrenda de sangre. Cuando se mata un animal por razones que no son alimentarias, principalmente cuando se mata un perro porque está muy enfermo y no puede recuperarse o porque se porta muy mal, o a un caballo enfermo, se dice «sacrificar» al animal. Cuando muere un pastor o una pastora, luego del velorio y del entierro, después de ocho días, se realiza un segundo entierro al lado de la casa y se «sacrifica» al perro del pastor para enterrarlo junto con un muñeco hecho con la ropa del muerto/a colocado sobre el animal, como si fueran el perro un caballo y el muñeco un jinetes.

El sacrificio para el entierro del pago puede consistir en las colas de los corderos cortadas según «el estilo» de Jasimaná, como se dice, aunque me comentaron que también se puede ofrecer a la Pachamama un feto de oveja o un cordero entero, sacrificado, bien floreado y chimpeado.

Como recién subrayamos, también en el día de las almas se destaca una lógica de ofrenda en forma de entierro, pero en este caso no se realiza un sacrificio, sino que al lado de los bienes alimentarios-rituales se ofrece comida cocinada de manera especial por cantidad y calidad, preparada a «la manera de antes».

«Los de antes» hace referencia a los antepasados cuya identidad y nombre son recordados, pero también los antepasados en general, en tanto que categoría indiferenciada, que corresponde también a los antiguos habitantes del lugar o, como dicen, a «los indios»6. Todos ellos, «los de antes», pueden volver por el día de la almas a visitar a la familia.

La lógica ritual en este caso es la de una ofrenda-convite: se trata de una visita, la llegada de seres queridos, esperados, mimados con manjares. Pero a dicha lógica del convite, basada en el encuentro entre vivos y muertos, se le sobrepone otra: la lógica de la separación. De hecho, vivos y muertos no «comparten»: los vivos no comen en la mesa de las almas, la comida se separa antes de ofrecerla y también en el momento de comer, cuando se separa el primer bocadillo. Las almas son esperadas y nombradas pero finalmente despachadas y hasta de prisa: los muertos que llegaron de visita a la casa no tienen que quedarse. Si durante el pago a la Tierra los miembros de la familia comen alrededor del agujero, «compartiendo» con la Pacha, dándole la mitad de lo que van a gustar, el 2 de noviembre se come

5 Sobre el perro que guía el alma del difunto en el viaje hacia el mundo de los muertos en el noroeste de Argentina, se puede ver por ejemplo Hoyos (1999), para el valle del Cajón, Catamarca.

6 Los pobladores de Jasimaná se reconocen como «indígenas» y consideran a «los indios» como los antiguos pobladores del lugar y sus antepasados. La categoría local de «indígena» define una posición de consciencia y lucha política y cultural, mientras que la categoría de «indio» guarda cierta ambigüedad: implica todavía una posición social subalterna pero también una nueva valoración de la diferencia cultural y de un pasado que fundamenta al presente indígena. 
después del entierro, cuando las almas han sido despachadas y supuestamente ya se han ido.

Ahora bien, ¿qué nos dicen estas, a veces, diferentes lógicas rituales y categorías de relación subrayadas, con respecto a los seres involucrados en los ritos y a sus relaciones recíprocas? Si el ritual produce y al mismo tiempo expresa relaciones, ¿de qué relaciones se trata? El análisis de las lógicas rituales puede ayudarnos a interpretar qué tipo de relación establecen los ritos descritos entre los seres involucrados. Por un lado, si las lógicas destacadas son diversas entre ellas, se podría suponer que las relaciones producidas por los rituales son de tipo diverso. Dichas diferencias relacionales implicarían diferencias entre los seres mismos, que serían distintos, aunque interrelacionados. Por otro lado, lógicas rituales parecidas, como la de la ofrenda en forma de entierro, indicarían un tipo de relación en cierta medida semejante y características compartidas por los seres no humanos involucrados, como las almas y la Pachamama en relación con los humanos que les ofrendan bienes a través del entierro. Entonces, ¿de qué seres se trata y en qué sentido son diferentes unos de otros?

\section{RELACIONES ENTRE SERES: CONTINUIDADES Y DIFERENCIAS ONTOLÓGICAS}

P. Descola, en el libro Par-delà nature et culture (2005) y en otros ensayos que sintetizan su teoría (2011), afirma que el rumbo de la Antropología es «describir, clasificar y tornar inteligibles las relaciones que los humanos establecen entre ellos y con los no humanos» (Descola, 2011: 87). Dichas relaciones son múltiples pero no infinitas, lo que hace posible identificar unos pocos «sistemas» de relaciones entre seres o «existentes», definidos como «ontologías» por el autor, a saber: el animismo, el totemismo, el analogismo y el naturalismo. Se trata de «sistemas de propiedades de los existentes que sirven de punto de anclaje de las formas cosmológicas, de los modelos de lazo social y de las teorías de la alteridad» (Descola, 2011: 87). Dichos sistemas radican en diferentes esquemas de percepción del medioambiente, incorporados por los individuos en el seno de su colectividad7, principalmente los de identificación y diferenciación de los existentes en relación a su fisicalidad e interioridad.

El sistema analógico, que el autor indica como típico, entre otros, de los mundos andinos, se basa en la percepción del medioambiente en tanto que «cosmo», es decir un universo único compuesto por existentes diferentes unos de otros tanto por su fisicalidad como por su interioridad, y generalmente formados ellos mismos por elementos diversos en equilibrio inestable. Estos seres, humanos y no-humanos, son interrelacionados por medio de analogías, similitudes, simpatías, correspondencias, resonancias e influencias recíprocas. Un ejemplo de sistema analógico es la «cadena de los existentes» que, a partir de la filosofía de Plotino, dio forma al pensamiento

7 Sin embargo, estos esquemas pueden también mezclarse entre ellos, hasta cierto punto. 
europeo por lo menos hasta el siglo XVIII. A lo largo de esta cadena los seres son relacionados entre sí por discontinuidades graduales que, a partir de cierto punto, se tornan diferencias y en algunos casos llegan a ser radicales.

Ahora bien, asumiendo que los rituales realizados por los pastores y pastoras de Jasimaná puedan corresponder a un sistema ontológico analógico, analizaremos y compararemos las relaciones entre los seres involucrados tanto en el pago como en el día de las almas, a partir de las categorías émicas y de las lógicas rituales destacadas.

El «hambre» de la Pachamama, a la que corresponde el «cuidado» de los humanos y el «dar de comer a la Tierra» por medio de una ofrenda-sacrificio, subraya el poder asombroso de la Pachamama con respecto al ser humano, y sugiere una relación de asimetría entre estos dos tipos de seres, la Pachamama y las personas, pese a las similitudes, a los espejismos y a la posibilidad de comunicación entre ellos (Bastien, 1985 [1978]). Se trataría, en definitiva, de entidades-agentes con características y posibilidades de acción recíproca bien diversas, lo que supone una diferencia o, tomando en cuenta el esquema analógico de la cadena de los existentes, una «distancia ontológica» entre ellos. De hecho, la Pachamama es una entidad muy poderosa, benévola pues brinda buenas cosechas de corderos y salud para la hacienda y para los miembros de la unidad doméstica, pero también es peligrosa para los animales y los humanos, ya que puede hacerlos caer, enfermar y también puede «comerlos». Sobre todo, la Tierra se vuelve peligrosa en agosto, cuando está «abierta», ya que en las regiones andinas las aperturas suponen la posibilidad de contacto e interacción entre seres diferentes y, por lo tanto, una amenaza física, pero también conceptual, para las personas (Fernández Juárez, 1996: 224-225). Además, los habitantes de Jasimaná dicen que la Pacha puede «castigar», hasta por faltas de las que, a lo mejor, uno no se dio cuenta en el momento. Analizando casos como este, Rösing (1994) introdujo el concepto de «deuda de ofrenda». A pesar de esto, la relación hacia la Pachamama no se basa en el miedo y el temor. Durante el pago se quiere «compartir» con ella que aprecia la alegría de la gente en esta ocasión y las personas suelen dirigirse a ella de una forma cariñosa y hasta tierna, Ilamándola «Pacha», «Pachita» o «Mamita». Según Fernández Juárez (1994: 57), en las regiones andinas aimaras, la ofrenda abundante es apercibida como «cariño» hacia la Pacha, y se considera que los dulces y bienes alimentarios ofrendados intentan satisfacer los «caprichos y debilidades culinarias» de la Pachamama.

Sin embargo, las lógicas rituales del pago nos indicarían una relación de diferencia radical entre la Pachamama, de un lado, y las personas con su hacienda, del otro. En cambio, las relaciones que se producen por el día de las almas marcan tanto una distancia como una cercanía entre personas y almas. Pese a la lógica de separación entre vivos y muertos, la ofrenda en tanto que convite implica el encuentro en la casa, es decir un sitio íntimo y doméstico: se trata de un acercamiento posible gracias a ciertas características comunes entre los seres involucrados. Al respecto, las categorías de «almas» y de «los de antes» sugieren cierta continuidad ontológica entre vivos y muertos. 
En primer lugar, las almas son tanto los muertos en general, como los parientes muertos. «Los de antes» son los antiguos pobladores del lugar y «los indios», pero al mismo tiempo son los antepasados: se trata de categorías indiferenciadas de difuntos y también de categorías de parentesco. Las almas de los parientes muertos son «familia» como los otros parientes vivos que participan en el rito. Además, al igual que en otros lugares de la macroárea andina8, en donde muchas de las categorías émicas de parentesco radican en las prácticas del compartir la cocina y la comida (Brush, 1977; Weismantel, 1988; Arnold ed., 1998), también en Jasimaná la familia y la unidad doméstica están asociadas al «comer juntos» y al «tener la cocina aparte». La cocina y la comida son respectivamente el sitio y el elemento central del día de las almas, lo que establece una asociación muy fuerte entre almas y parientes.

En segundo lugar, un «alma» no solo corresponde al difunto, sino que también es parte de la persona viva. El alma coincide con la conciencia individual y con el nombre de la persona. Por ejemplo, a causa del «susto», es decir de un trauma debido a una caída o a un encuentro inesperado con animales peligrosos o con espíritus y duendes 9 que andan por los caminos, la persona puede perder su alma, quedando enferma, nerviosa hasta la locura o deprimida, con consecuencias muy graves. Para curarse, necesita de un médico campesino que pueda «llamar» ritualmente al alma perdida.

Entonces, si por un lado la Pachamama y la persona se relacionan ritualmente como seres ontológicamente bien diferentes, por el otro lado, almas y personas pueden ser parientes entre sí, además de que toda persona tiene una alma (es decir, conciencia y nombre).

Sin embargo, las almas de los muertos, sobre todo si fallecieron de forma violenta, pueden quedarse por un tiempo en la tierra (mientras que las demás solo vuelven por el día de las almas) y asustar a las personas a lo largo de los caminos, igual que los espíritus y los duendes. En definitiva, los muertos también son peligrosos: por eso hay que realizar el entierro de comida en el patio, afuera de la casa y despachar de prisa a las almas al mediodía del 2 de noviembre.

Además, la lógica compartida de la ofrenda en forma de entierro, que se realiza tanto por el pago a la Tierra como por el día de las almas, podría sugerir una asociación entre muertos y Pachamama, o sea, la existencia de propiedades comunes. De hecho, varios autores subrayaron cómo en diferentes lugares de la macroárea andina, los muertos, principalmente los antepasados, ayudan a los vivos en la producción agrícola, permitiendo a las plantas brotar desde la tierra y favoreciendo su crecimiento (Berg, 1992; Arnold et al., 2007; etc.), garantizando así una buena cosecha a sus descendientes y parientes y, en general, a los vivos ${ }^{10}$. Esta relación con los cultivos y la cosecha marcaría una similitud entre las almas y la Pachamama.

8 Ecuador, Perú, Bolivia, norte de Chile y noroeste de Argentina.

9 Los duendes son generalmente espíritus de criaturas abortadas (Colombres, 1986: 136).

10 Por ejemplo, con respecto a las comunidades aimaras, H. van den Berg (1992: 295) escribe: «Estos difuntos forman un grupo que, por un lado, infunde temor, mientras que, por otro lado, es 
Finalmente, en la literatura antropológica se hallan sugerentes asociaciones entre los antepasados, los antigales (es decir, los antiguos poblados), las wakas (rocas y también antiguas tumbas) y los apus o achachilas (cerros sagrados, en quechua y en aimara, respectivamente), aunque estos generalmente se consideran diferentes de la Pachamama (Berg, 1992; Spedding, 2008). En Jasimaná también se establece una asociación entre los muertos, los antepasados y los antiguos pobladores llamados «los indios», por medio de la categoría de «los de antes», pero las propiedades supuestamente comunes a los muertos y a la Pachamama no son establecidas de manera explícita; sin embargo, hay diferencias importantes entre estos tipos de seres.

\section{4. «CONVIDAR» Y «COMPARTIR»: PRÁCTICAS Y CATEGORÍAS DE ACERCAMIENTO ONTOLÓGICO}

Las diferencias ontológicas entre seres son muy profundas en el caso de la Pachamama y las personas con su hacienda, relacionadas por medio del pago a la Tierra, mientras que parecen menos radicales en el caso de las almas de los muertos, las almas de los parientes muertos, los parientes vivos, además de las almas de los vivos, relacionadas entre ellas por el rito del día de las almas. Sin embargo, ciertas prácticas locales y categorías émicas vuelven un poco más ambiguas dichas diferencias entre personas, almas y Pachamama.

En primer lugar, se trata de la práctica y de la categoría del «convidar»: a lo largo de los caminos, cuando se cruzan personas se les convida hojas de coca, pero también se convida a la Pacha donde se hallan apachetas, dándole bebidas y coca, aunque es posible convidarla enterrando coca o soplando las hojas en el aire en cualquier sitio. Como ya señalamos, por el día de las almas se «Convida» comida y bienes alimentarios-rituales a las almas, así como se hace con los demás parientes. En el caso del pago, si bien no se trata tanto de un convite sino de una forma de sacrificio, esto es una manera de «compartir» con la Pachamama, brindándole una parte de lo que uno mismo goza.

En segundo lugar, hay categorías y prácticas de «personificación» que vuelven tanto a las almas como a la Pachamama parecidas a las personas. En la noche del 1 de noviembre, por ejemplo, las almas de ciertos muertos son nombradas, dándoles de esta manera el atributo de las personas, puesto que el nombre coincide con la conciencia individual. Con respecto a la Pachamama, según muchos relatos de los pastores y pastoras de Jasimaná, ella puede aparecer bajo la forma de espíritu, como una viejita o una mujer que anda por el cerro con mucha hacienda (en el caso de la puna de Catamarca, véase por ejemplo García \& Rolandi, 2000).

considerado como un grupo que está estrechamente relacionado con las vicisitudes de los vivientes. Ellos forman parte de la comunidad de los vivos: hay una convivencia entre los vivientes y las almas de los parientes difuntos. Estos últimos necesitan de la ayuda de los vivientes, mientras que, al mismo tiempo, pueden prestar servicios a los vivos. Al revés es lo mismo: los vivos necesitan de la colaboración de las almas de los difuntos, por ejemplo para conseguir buenas cosechas, y a su vez prestan servicios a los difuntos en lo que respecta a su marcha hacia una existencia tranquila». 


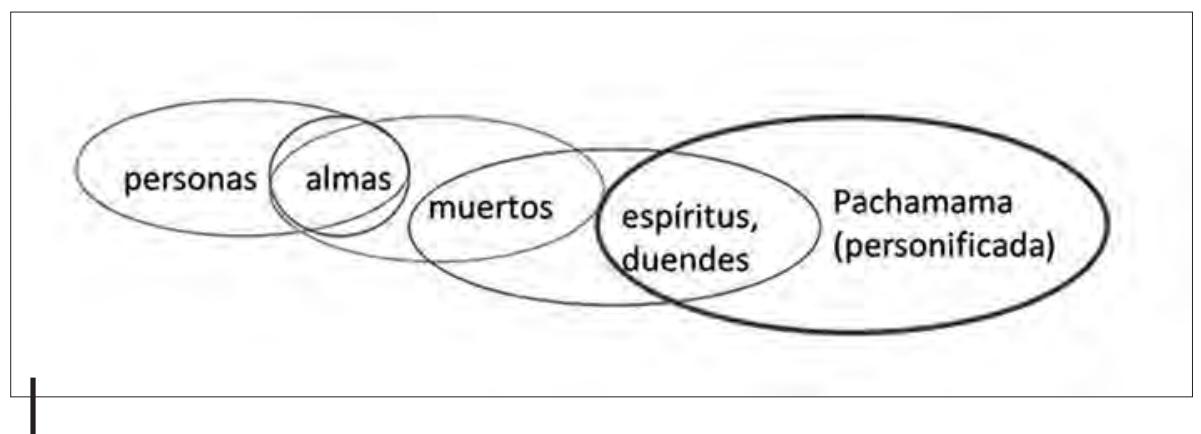

Figura 3 - Ejemplo de cadena analógica de seres

(c) Daniela Salvucci

Por lo tanto, en el altiplano andino de Jasimaná, pese a la distancia y diferencia ontológica evidente en las relaciones producidas por los dos ritos analizados, las prácticas y las categorías de relación del convidar, del compartir, del parentesco y las posibles personificaciones de los difuntos y de la Pachamama, permiten un acercamiento ontológico entre seres humanos y no-humanos, como las almas, las personas y la Pachamama. Este acercamiento reduce, en ciertas ocasiones, la distancia entre ellos y permite a través de esta proximidad recorrer la cadena de los existentes, mirar en el vértigo de las analogías y reconocer en el espejo al Otro, hallar por un momento el hilo que lo une todo.

\section{Referencias citadas}

ARNOLD, D. (ed.), 1998 - Gente de carne y hueso: las tramas de parentesco en los Andes, Tomo II, 608 pp.; La Paz: Instituto de Lengua y Cultura Aymara, Centre for Indigenous American Studies and Exchange.

ARNOLD, D., YAPITA, J. \& ESPEJO AYCA, E., 2007 - Hilos sueltos: los Andes desde el textil, 428 pp.; La Paz: Instituto de Lengua y Cultura Aymara, Plural editores.

BASTIEN, J. W., 1985 [1978] - Mountain of the Condor. Metaphor and Ritual in an Andean Ayllu, xxv + 227 pp.; Illinois: Waveland Press.

BERG, H. van den, 1992 - Religión aymara. In: La cosmovisión aymara (H. van den Berg \& N. Shiffers, eds.): 291-308; La Paz: Universidad Católica Boliviana, Editorial Hisbol.

BRUSH, S. B., 1977 - Mountain, Field and Family: The Economy and Human Ecology of an Andean Valley, xiv + 199 pp.; Pennsylvania: University of Pennsylvania Press.

BUGALLO, L., 2014 - Flores para el ganado. Una concepción puneña del multiplico (Puna de Jujuy, Argentina). In: Comprender los rituales ganaderos en los Andes y más allá. Etnografías de lidias, herranzas y arrierías, BAS 51 (J. J. Rivera Andía, ed.): 311-363; Aachen: Schaker Verlag. 
Lógicas rituales y categorías de relación entre seres en la Argentina andina

COLOMBRES, A., 1986 - Seres sobrenaturales de la cultura popular argentina, 203 pp.; Buenos Aires: Ediciones del Sol.

DESCOLA, P., 2005 - Par-delà nature et culture, 640 pp.; París: Édition Gallimard.

DESCOLA, P., 2011 - Más allá de la naturaleza y de la cultura. In: Cultura y Naturaleza (L. Montenegro Martínez, ed.): 75-97; Bogotá: Jardín Botánico de Bogotá, José Celestino Mutis.

FERNÁNDEZ JUÁREZ, G., 1994 - Tinku y Taypi: dos recursos culinarios pertinentes en las ofrendas aymaras a la Pachamama. Anthropológica, 11: 49-78.

FERNÁNDEZ JUÁREZ, G., 1996 - El mundo «abierto»: agosto y Semana Santa en las celebraciones rituales aymaras. Revista Española de Antropología Americana, 26: 205-229.

FLORES OCHOA, J., 1977 - Enqa, enqaychu, illa y khuya rumi. In: Pastores de puna. Uywamichiq punarunakuna (Flores Ochoa, ed.): 211-237; Lima: Instituto de Estudios Peruanos.

GARCÍA, S. P. \& ROLANDI, D., 2000 - Relatos y ritual referidos a la Pachamama en Antofagasta de la Sierra, puna meridional argentina. Relaciones de la Sociedad Argentina de Antropología, XXV: 7-25; Buenos Aires.

GEERTZ, C., 1973 - The Interpretation of Cultures, ix + 470 pp.; New York: Basic Books.

GIL MONTERO, R., 2004 - Caravaneros y trashumantes en los Andes Meridionales. Población y familia indígena en la puna de Jujuy 1770-1870, 320 pp.; Lima: Instituto de Estudios Peruanos.

HARVEY, P., 1998 - Los "hechos naturales" de parentesco y género en un contexto andino. In: Gente de carne y hueso: las tramas de parentesco en los Andes, Tomo II (D. Arnold, ed.): 69-82; La Paz: ILCA, CIASE.

HOYOS, M. de, 1999 - Saliendo del cajón por el río Jordán: costumbres funerarias del valle del Cajón, Catamarca, Argentina. In: Los vivos y los muertos. Duelo y Ritual Mortuorio en los Andes (J. Van Kessel, ed.): 177-183; Iquique: Instituto para el Estudio de la Cultura y Tecnología Andina.

JIMÉNEZ SÁNCHEZ-MARISCAL, D., 2014 - Palimpsesto cultural y palingenesia del pensamiento: una aportación andina a la filosofía de la religión, 372 pp.; Madrid: Universidad Complutense. Tesis doctoral.

RÖSING, I., 1994 - La Deuda de Ofrenda: un concepto central de la religión andina. Revista Andina, 12 (1): 191-216.

SALVUCCI, D., 2013 - Culture della parentela nella valle e altopiano Calchaquí, Salta, Argentina andina, 293 pp.; Siena: Università degli Studi di Siena. Tesis doctoral.

SPEDDING, A. P., 2008 - Religión en los Andes. Extirpación de idolatrías y modernidad de la fe andina, 200 pp.; La Paz: Instituto Superior Ecuménico Andino de Teología.

WEISMANTEL, M. J., 1988 - Food, Gender and Poverty in the Ecuadorian Andes, xii + 234 pp.; Philadelphia: University of Pennsylvania Press. 